\title{
Development of a Training Program for Handling Hazardous Drugs
}

\author{
Donna M M Woloschuk, Wendy Simoens, Clayton Hayes, Lorraine Woods, Jill Krevesky, \\ and Florence Mendelson
}

\section{INTRODUCTION}

$\mathrm{T}$ o successfully implement large-scale change initiatives, such as new safe-work procedures, supervisors need to consider how much education is required and who the target audiences are. Plans need to take into account the resources required for initial implementation, as well as those needed to sustain training afterward. ${ }^{1,2}$

In 2002, responding to major changes in workplace safety and health legislation, managers in the Winnipeg Regional Health Authority (WRHA) Pharmacy Program began a systematic review of drug and nondrug occupational hazards. Processes used for receipt, storage, preparation, distribution, administration, and disposal of drugs, as well as chemicals used for extemporaneous compounding, were also evaluated. One major initiative arising from these reviews, the development of a regional program for safe handling of hazardous drugs, has been described previously. ${ }^{3}$ Staff training was one component of the implementation plan. This report describes the development of and enhancements to the WRHA Pharmacy Program's Safe Handling of Hazardous Drugs Training Program over the period 2008 to 2012 .

\section{DESCRIPTION OF THE PROJECT}

The goal of this project was to create an effective, accessible, sustainable, multifaceted training program for the handling of hazardous drugs that was suitable for pharmacy staff but that also might be easily adapted for other health workers. The training program also needed to include a mechanism to ensure that staff members were advised, on a continuing basis after initial implementation, about known or reasonably foreseeable risks to safety and health arising from hazardous drugs used in their work areas. The WRHA Pharmacy Program Practice Development team, comprising 2.5 full-time equivalent staff, assumed responsibility to develop and coordinate delivery of education for approximately 360 full-time equivalent union- ized pharmacists, pharmacy residents, and technicians at 8 facilities. When literature and grey literature searches revealed no existing training programs for the safe handling of hazardous drugs, the Practice Development team developed a training program to support policy implementation by the pharmacy's Hazardous Drugs Handling Committee (HDHC). The team's focus was training related to new safe-work procedures using the correct personal protective equipment (PPE), rather than general education about safe handling of hazardous drugs.

\section{Needs Assessment}

A needs assessment was conducted using an open questionnaire and interviews with members of the HDHC and the pharmacy leadership team. Analysis of those data revealed 3 training scenarios and associated logistic requirements.

Training of existing staff: Training of existing staff had to occur quickly, use existing resources, involve only 2 instructors, and have minimal development costs. Day-shift staff members requested face-to-face inservice sessions, whereas evening and night staff requested online learning. ${ }^{4,5}$ Instruction was intended to focus on changes to procedures and use of $\mathrm{HDHC}$ job aids (concise, easily accessed information that helps workers to perform a task correctly and completely).

Training of subsequently hired new staff: Education of subsequently hired new staff focused on the requirements that the educational material be easily accessible to staff, integrate seamlessly into existing staff orientation processes, use available equipment, avoid the need for specialized trainers, and be easily revised for use by other health care workers. ${ }^{4,6}$

Updating of existing staff: To ensure up-to-date knowledge of current safety practices, refresher training had to be effective and easily accessible to staff, had to have low delivery and maintenance costs, had to allow evaluation of all pharmacy staff, and had to provide evidence of ongoing training. 


\section{Design and Implementation}

To meet the needs that had been identified, we first developed a "Safe Handling of Hazardous Medications" inservice module and a self-learning package (Table 1) to serve as the main training tools for existing staff. The self-learning package was also designed to serve as the main training tool for future staff. In preparing the self-learning package, Practice Development staff reviewed existing HDHC resources (Box 1) and determined what information would be incorporated. The content of the package focused on use, interpretation, or application of information already available in $\mathrm{HDHC}$ resources. The content was prepared at a grade 10 reading level using a standard template. Questions were added to encourage practice using job aids, as were scenarios intended to prompt thinking about and reading of the resources. The scenarios were suited to pharmacist and pharmacy technician job tasks. The self-learning package and all resources were made available on a purpose-built intranet web page. The inservice module, which was used only for initial training of existing staff (day shift), was delivered as multiple 90-min sessions over the period February to May 2009. The content included core concepts from the self-learning package $(40 \mathrm{~min})$, team-specific procedure changes (40 min), and a question period (10 min). Inservice attendance logs provided evidence of training. Staff members who could not attend the inservice sessions were required to read the self-learning package. Subsequently hired new staff members were required to review the self-learning package within the first 90 days of employment. Existing staff and subsequently hired new staff were not required to complete a quiz following the inservice module or review of the selflearning package.
For intermittent refresher training, the Practice Development team created a multiple-choice refresher quiz and a mock spill drill that would be completed annually by all pharmacy staff. The refresher quiz was developed and field-tested in 2008 but was not fully implemented until November 2010. The quiz (70\% multiple-choice questions and 30\% matching or short answer questions) probed staff members' knowledge about safe handling procedures. Subject areas with highest risk if performed incorrectly were assigned the greatest weight on the quiz. The quiz blueprint covered theory related to hazardous drugs $(15 \%)$, use of wall charts $(50 \%)$, procedures and guidelines $(10 \%)$, handling of compromised enteral dosage forms

\section{Box 1. Resources Available for Education about Safe Handling of Hazardous Drugs}

Pharmacy Safe Handling of Hazardous Drugs wall chart Cytotoxic and Non-Cytotoxic Hazardous Drugs List Enteral Compromised Dosage Form List

Spill Management Algorithm

Algorithm for the Safe Handling of New Drugs

Procedures for Preparing Cytotoxic Compromised, Cytotoxic

Oral Intact, and Non-Cytotoxic Hazardous Oral Intact

Dosage Forms

Guidelines for Injectable Non-Cytotoxic Hazardous Drugs,

Oral Cytotoxic, and Non-Cytotoxic Products

Steps for Choosing Enteral Compromised Dosage Forms

Dissolve and Dose Instructions for Nurses

Crush, Dissolve, and Dose Instructions for Nurses

Powder or Powder from a Capsule Instructions for Nurses

WRHA Safe Handling of Cytotoxic Drugs - Introduction

Digital Video Disc (DVD)

WRHA Safe Handling of Cytotoxic Drugs - Preparation in

Pharmacy (DVD)

WRHA = Winnipeg Regional Health Authority.

\section{Table 1. Content of Self-Learning Package for Safe Handling of Potentially Hazardous Drugs*}

\begin{tabular}{|c|c|}
\hline Module & Content \\
\hline $\begin{array}{l}\text { 1: What are hazardous drugs? Why must I use caution when } \\
\text { handling certain drugs? }\end{array}$ & $\begin{array}{l}\text { - Terms to know when handling potentially hazardous drugs } \\
\text { - Potentially hazardous drugs and occupational health risks } \\
\text { - Conditions that increase exposure risk and harm potential }\end{array}$ \\
\hline $\begin{array}{l}\text { 2: What type of precautions should I use when handling } \\
\text { hazardous drugs? }\end{array}$ & $\begin{array}{l}\text { - Make a C.H.O.I.C.E.† to handle hazardous drugs safely } \\
\text { - Correctly interpret the Cytotoxic \& Non Cytotoxic Hazardous } \\
\text { Drugs chart } \\
\text { - Correctly interpret the Safe Handling of Drugs chart }\end{array}$ \\
\hline $\begin{array}{l}\text { 3: Interpreting guidelines, procedures and policies, and nursing } \\
\text { instructions for enteral administration of hazardous drugs }\end{array}$ & $\begin{array}{l}\text { - Deciding which resource to use; how to interpret and apply } \\
\text { information from available resources } \\
\text { - What is enteral drug administration? } \\
\text { - How to safely compromise a dosage form to facilitate enteral } \\
\text { administration }\end{array}$ \\
\hline 4: How to clean up a spill of a hazardous drug & $\begin{array}{l}\text { - Classifying spills } \\
\text { - What to do if you come in contact with a spill } \\
\text { - How do you properly clean up a chemo spill? }\end{array}$ \\
\hline
\end{tabular}

*(C) 2009 Winnipeg Regional Health Authority Pharmacy Program. Reproduced with permission.

†C.H.O.I.C.E. is a memory aid, referring to the following questions? Is the medication a Cytotoxic drug? Is the medication a non-cytotoxic Hazardous drug? Is the medication an intact Oral dosage form? Is the medication an Injectable product? Is the dosage form Compromised? What Effect do these answers have on how I should handle the drug? 
(10\%), and handling of spills (15\%). A question bank was developed, and quizzes with 20 questions (for a total of 20 points) were created. The open-book, self-scheduled, unsupervised quizzes had no time limit for completion; the minimum passing score was $90 \%$, and any incomplete or illegible responses were to be scored as incorrect. After the initial field test, completed quiz papers were returned to administrative assistants, who marked them using an answer key, made the result available to each staff member, and sent all results to a member of the Practice Development team. This team member analyzed the results to identify patterns of incorrect response that might indicate a need for revisions to the self-learning package. Staff members who did not achieve the passing score were asked to redo the quiz until they achieved a passing score.

The Hazardous Drugs Mock Spill Drill Toolkit for Managers was designed and implemented in 2012. Literature and grey literature searches yielded no resources for mock spills of hazardous drugs; therefore, guides for other emergency drills were analyzed. Drill requirements were found to include a notification process, a statement of drill frequency, responsibilities of the staff involved, instructions for conducting a drill, a documentation checklist, and conduct of a post-drill debriefing session. Simple instructions and resources for each phase of the drill, including communication and documentation tools, were gathered into a toolkit (Box 2). Black and white graphic images facilitated conduct of a mock spill. Forms enabled quick recording of staff responses (Table 2).

\section{Evaluation}

Before the self-learning package was implemented, a convenience sample of 12 volunteer pharmacy staff members ( 8

\section{Box 2. Content of Hazardous Drugs Mock Spill Drill Toolkit for Managers}

\begin{tabular}{l} 
How to access resources on handling hazardous drugs \\
\hline Instructions for conducting a mock spill drill \\
Forms \\
Spill drill checklist \\
Spill drill follow-up form
\end{tabular}

\section{Communication tools}

Sample staff notification (e-mail)

Conducting the debriefing session: tips and sample script

Mock spill graphics

Small spill (liquid)

Small spill (intact tablets)

Large spill (liquid)

Large spill (liquid with broken glass)

Large spill (intact tablets)

Large spill (powder with broken glass) pharmacists and 4 technicians) reviewed the initial version of the package and answered an evaluation survey (during paid work time, to facilitate complete participation). The Practice Deveopment technician analyzed the collated results using qualitative description to identify themes. ${ }^{7.8}$ Time to complete the initial version of the self-learning package ranged from 15 to $59 \mathrm{~min}$. Despite most reviewers agreeing with the format and content of the self-learning package, there were some suggestions for improvement: provide step-by-step information about handling of cytotoxic drug spills; indicate where to find the HDHC resources; provide a better definition of "cytotoxic", indicating cell death, to convey the potential for harm; and provide information about how the HDHC job aids are updated and maintained.

Concerns raised by new staff, trainers, and managers through to the end of 2009 made it clear that the HDHC resources required significant revision. Feedback identified the need for clarity of complex information in existing HDHC resources, revision of the spill-handling policy, practical training about how to handle spills, and description of procedures for drugs not listed in the existing hazardous drug list. ${ }^{9}$ Over the subsequent 3 years, the HDHC revised the hazardous drugs list and modified the spill-handling procedure, which led to changes to the self-learning package. Similarly, feedback from staff regarding the content of the face-to-face inservice sessions resulted in revision of inservice and self-learning package content. Finally, an audit done for another project identified that incorrect PPE was being used in some pharmacy areas. When the correct PPE became available in pharmacy service areas, staff members performed better on quiz questions involving PPE.

The initial field test of the refresher quiz was conducted in conjunction with the field test of the self-learning package in April 2008. The Practice Development technician scored the quizzes, documented total marking time, and analyzed response patterns. The quiz could be completed by most staff in less than $20 \mathrm{~min}$ and could be scored in $2-3 \mathrm{~min}$ by a pharmacy administrative assistant. Evaluation of test performance identified ambiguous questions, which were removed, as well as the need to simplify wording and eliminate duplication. Appropriate revisions to the quiz were completed, and a revised version has been administered annually, from 2010 forward, to all pharmacy staff, excluding administrative assistants. In 2012, the WRHA Pharmacy Program approved a competency assessment policy for handling hazardous drugs.

An initial mock spill drill confirmed the appropriateness and clarity of content of the spill drill toolkit and provided an estimate of the time necessary for preparation, completion, documentation, and debriefing for subsequent mock spill drills. Subsequent drills have revealed opportunities for further clarification and simplification of instructions for handling spills. 


\section{Table 2. Sample Documentation Checklist for a Spill Drill}

Checklist Item

Cleanup procedures initiated immediately

Spill isolated (signs placed if necessary)

Staff member notifies other staff in area of spill, calls for help if required

If spill occurs in sterile compounding room/area, all staff follow proper aseptic garb/

supplemental hand-washing procedures

Staff member(s) don personal protective equipment (PPE) appropriate for size and

nature of spill (small or large)

Small/easily contained spill $(<25 \mathrm{~mL})$

- 2 pairs of gloves (interior gloves under cuffs of gown, exterior gloves are chemo

gloves and are pulled over cuffs of gown)

- chemo gown, mask (depending on nature of spill)

Large contained or uncontained spill (> $25 \mathrm{~mL})$

- 2 pairs of gloves (interior gloves under cuffs of gown, exterior gloves are chemo gloves

and are pulled over cuffs of gown)

- chemo gown and mask

- goggles and foot covers from spill kit

Clean-up appropriate for size and nature of spill (small or large)

Small/easily contained spill $(<25 \mathrm{~mL})$

- cover liquids with absorbent pad, plastic side up (absorbent side down) till completely

absorbed, avoid splashing

- remove tablets with paper towels, gloved hands

- wash area: detergent + water + paper towel 3 times (rinse with water after each wash)

Large contained or uncontained spill (> $25 \mathrm{~mL})$

- find and open Chemo Spill Kit, place signage

- prevent spreading by gently covering with absorbent pad, plastic side up (absorbent side down)

- be careful not to create aerosols: slow and careful movements to minimize large air drafts (wait for aerosols to settle if necessary)

- pick up powder with moistened absorbent material (paper towel)

- when sharps or broken objects are present, use scoop, not hands

- wash area: detergent + water + paper towel 3 times (rinse with water after each wash)

- dry area with remaining plastic-backed pads

Waste and PPE disposed of in correct and labellled cytotoxic waste container

Soiled clothing isolated in plastic bag for separate laundering

Nondisposable equipment washed with detergent and water

Hands (and/or other exposed skin) washed thoroughly with soap and water

Be aware to call housekeeping for terminal cleaning (for drill, do not place call)

Spill kits reordered

Notify manager/documentation forms completed

First aid given immediately if direct contact with spill

Eyes: flush with copious amounts of water or normal saline for 15 minutes

Skin: remove contaminated clothing and wash area with soap and running water for at

least 15 minutes (in case of skin puncture, also squeeze puncture site to encourage bleeding)

Pharmacy: __ Service Area: __ Date: __ Time

Names of employee(s) present:

Type of spill (large, small, liquid, tablet, powder, contained, uncontained, broken glass)

Name of manager who conducted drill:

Fax completed form to: 


\section{IMPLICATIONS FOR PRACTICE}

It is vital, from a workplace health and safety standpoint, to ensure the safety of all staff members who handle hazardous materials. This experience has shown that improving worker safety requires not only a policy with associated procedures, but also a comprehensive training program to ensure initial and ongoing use of high-quality resources (e.g., job aids) by frontline staff members. When implementing an initiative of this complexity and magnitude, considerable time should be devoted to planning how education will help to successfully bridge the gap between policy and actual practice. An evaluation and ongoing audit of the availability of appropriate PPE is also vital to ensure that hazardous drugs will be handled properly. Without adequate forethought for a training program that incorporates quality assurance processes, there is a risk that front-line staff members will not benefit from carefully considered workplace safety precautions. The WRHA Pharmacy Program now has a full complement of easily maintained and administered training materials that help to ensure the ongoing safety of pharmacy staff members who handle cytotoxic and non-cytotoxic hazardous medications. Assuring staff safety has been made easier by providing standardized initial training at the time of hiring, supplemented by intermittent refresher quizzes and mock spills, as well as maintenance of training records for all staff members. Total training time is less than $45 \mathrm{~min}$ for newly hired staff. Existing staff require approximately 20 min annually to complete a refresher quiz that takes an administrative assistant less than $3 \mathrm{~min}$ to score. Staff members also participate in a spill drill of $30 \mathrm{~min}$ duration every 12 to 18 months. This investment in staff training has been easily sustained since 2010 .

This experience has also demonstrated the importance of field-testing new training materials and policy-related resources. Content experts worked with an experienced instructional designer to create the training materials; however, all items required modification after feedback from front-line staff members or managers. A high degree of collaboration between HDHC, the WRHA committee responsible for the hazardous drugs handling policy, and the Practice Development team ensured that feedback was incorporated into new or revised HDHC resources and that release of new resources or policy was aligned with release of revised training materials.

The Practice Development team purposely developed training tools that could be easily adapted for use by other service providers in the WRHA. This has proven fruitful as, effective 2012, the inservice presentation, self-learning package, and spill drill toolkit have been adapted to train nursing staff across the health region. Adoption of the pharmacy Safe Handling of Hazardous Drugs Training Program for regionwide training of nursing personnel attests to the quality of the training program and the medication safety leadership role of the WRHA Pharmacy Program. Interested readers can order the self-learning package, spill drill toolkit, and HDHC resources (including drug lists and wall charts) by contacting Suzanne Bekker (sbekker@wrha.mb.ca).

\section{References}

1. Labesse ME. Terms of reference for training needs analysis: continuing education component. Quebec (QC): Government of Quebec, Institut national de santé publique du Québec; 2008 [cited 2012 Jul 20]. Available from: www.inspq.qc.ca/pdf/publications/884_Cadre_reference_ang.pdf

2. Training for improved performance. Unit 1: Needs assessment. Athabasca (AB): Athabasca University; 1990. pp. 9-34.

3. Sproll B, Milan H, Caligiuri C, Dyck S, Rosenthal B, Raymond CB. Development and implementation of a regional program for the safe handling of hazardous drugs by hospital pharmacies. Can J Hosp Pharm. 2012;65(3):223-8.

4. Woloschuk DM, Raymond CB. Multihospital collaborative orientation program for new pharmacy employees. Am J Health Syst Pharm. 2010; 67(2):118-21.

5. Krevesky JS, Raymond CB, Woloschuk DMM. Continuing professional development for pharmacy technicians: start of an evolution? Can Pharm J. 2012;145(3):120-2.

6. Raymond CB, Woloschuk DMM. Prior learning assessments in a professional workplace for practising pharmacists and technicians. Can Pharm J. 2011; 144(4):192-7.

7. Sandelowski M. Whatever happened to qualitative description? Res Nurs Health. 2000;23(4):334-40.

8. Patton MQ. Analysis, interpretation, and reporting. In: Laughton CD, Axelsen $\mathrm{DE}$, Peterson K, editors. Qualitative research and evaluation methods. 3rd ed. Thousand Oaks (CA): Sage Publications; 2002.

9. WRHA safe handling of hazardous medications policy 110.160.010. In: WRHA corporate policy manual. Winnipeg (MB): Winnipeg Regional Health Authority; 2012 Jul.

Donna M M Woloschuk, BSP, PharmD, MEd(Distance), FCSHP, is Regional Pharmacy Manager, Educational Services, with the Winnipeg Regional Health Authority Pharmacy Program, Winnipeg, Manitoba.

Wendy Simoens, BScPharm, is a Drug Distribution Systems Support Pharmacist with the Winnipeg Regional Health Authority Pharmacy Program, Winnipeg, Manitoba.

Clayton Hayes, BBA, is Pharmacy Technician Manager, Health Sciences Centre Site, Winnipeg Regional Health Authority Pharmacy Program, Winnipeg, Manitoba.

Lorraine Woods, BSCPharm, ACPR, CACE, is Education Coordinator with the Winnipeg Regional Health Authority Pharmacy Program, Winnipeg, Manitoba.

Jill Krevesky, BA, is Pharmacy Technician Manager, St Boniface Hospital, Winnipeg, Manitoba.

Florence Mendelson, BScPharm, is a Staff Pharmacist with the Winnipeg Regional Health Authority Pharmacy Program, Winnipeg, Manitoba.

Competing interests: This project received the 2012/2013 Safe Medication Best Practices Award from the Canadian Society of Hospital Pharmacists. Donna Woloschuk has served as a consultant for the Canadian Pharmacists Association (for work outside the scope of this article) and has received payment from Capital Health (Halifax), the Canadian Society of Hospital Pharmacists, and the Canadian Pharmacists Association for development of educational presentations.

\section{Address correspondence to:}

Dr Donna M M Woloschuk

Winnipeg Regional Health Authority Pharmacy Program

Deer Lodge Centre North Pavilion Room N245

2109 Portage Avenue

Winnipeg MB R3J OL3

e-mail: dwoloschuk@hsc.mb.ca 